Check for updates

Cite this: RSC Adv., 2017, 7, 43521

\title{
Adsorption sensitivity of graphane decorated with B, N, S, and Al towards HCN: a first-principles study
}

\author{
Qingxiao Zhou, (D) *ac Weiwei Ju, ${ }^{a}$ Xiangying Su, ${ }^{a}$ Yongliang Yong, ${ }^{a}$ Xiaohong $\mathrm{Li}^{a}{ }^{a}$ \\ Zhibing $\mathrm{Fu}^{\mathrm{b}}$ and Chaoyang Wang ${ }^{\mathrm{b}}$
}

The geometric structure, adsorption energy, electronic structure, and magnetic properties of hydrogenated graphene (graphane) with the adsorption of a $\mathrm{HCN}$ molecule were investigated by first-principles calculations. Compared with graphane, the adsorption of $\mathrm{HCN}$ on $\mathrm{H}$-vacancy defected graphane (VHG) exhibited higher stability, which implied that the $\mathrm{H}$-vacancy improved the sensitivity of graphane. However, the small adsorption energies and large bond distance indicated that the weak adsorption of a HCN molecule on the graphane and VHG substrates was due to physisorption. By introducing dopants (B, N, S, and Al), the activity of graphane was significantly improved. The adsorption of HCN changed to chemisorption on the graphane with dopants. Meanwhile, the opening of band gaps by HCN adsorption can be used as an electronic signal to detect HCN gas. Interestingly, the spin polarized density of states (PDOS) results suggested that the adsorption of HCN on VHG and S-doped VHG exhibited magnetic character and half-metallicity behavior. These results could provide useful information to design gas sensors for $\mathrm{HCN}$ or spintronic devices based on graphane.

Received 3rd August 2017

Accepted 5th September 2017

DOI: $10.1039 / \mathrm{c} 7 \mathrm{ra0} 8579 f$

rsc.li/rsc-advances been successfully attempted to improve the interaction between the graphene and absorbents. In this regard, graphane is a promising compound, which was first experimentally prepared by attaching hydrogen atoms on the graphene in 2009. ${ }^{17}$

In graphane, the full hydrogenation of graphene leads the $\mathrm{sp}^{2}$ hybridization of $\mathrm{C}-\mathrm{C}$ bond in graphene changed to $\mathrm{sp}^{3}$ hybridization in graphane. Graphane has been studied in recent past due to its unique electronic and magnetic properties. ${ }^{\mathbf{1 8 - 2 2}}$ Although the highly mechanical strength and wide band-gap of graphane add to its excellence in applications, the bonds $(\mathrm{C}-\mathrm{C}$ and $\mathrm{C}-\mathrm{H}$ bonds) formed in graphane cause weak activity to adsorbents. However, the sensitivity of graphane can be significantly improved by introducing of $\mathrm{H}$-vacancy, which also induced magnetism in graphane. ${ }^{23-28}$ Berashevich et al. investigated the influence of adsorbates on the electronic and magnetic properties of graphane, and they found that $\mathrm{H}$ vacancies significantly improved the chemical reactivity of graphane to environment. ${ }^{23}$ Islam et al. investigated the sensing aptitude of graphane by crafting with H-vacancy and dopants, and the results suggested that interaction of graphane towards $\mathrm{CH}_{4}$ gas molecules was enhanced by introducing $\mathrm{H}$-defect and dopants. ${ }^{29}$ Therefore, it is worth investigating the sensitivity to gas molecules of graphane with defects and dopants.

In this work, we systematically investigated the geometry, adsorption stability, electronic and magnetic properties of HCN adsorption on graphane. The modified graphane substrates considered here were pure, H-defected, and doped graphane, and the dopants were B, N, S and Al. We hope our study provides
${ }^{a}$ College of Physics and Engineering, Henan University of Science and Technology, Luoyang 471023, People's Republic of China. E-mail: zqxhaust@163.com; Tel: +86 18780539390

${ }^{b}$ Research Center of Laser Fusion, China Academy of Engineering Physics, Mianyang 621900, People's Republic of China

${ }^{c}$ College of Physical Science and Technology, Sichuan University, Chengdu 610065, People's Republic of China 
useful information to the application based on graphane for gas sensing.

\section{Computational methods and model}

All the DFT calculations were performed by the $\mathrm{Dmol}^{3}$ code. $^{30}$ We used the generalized gradient approximation (GGA) for exchange-correlation functional, as described by PerdewBurke-Ernzerhof (PBE). ${ }^{31}$ Basiuk et al. reported that PBE functional along with Grimme dispersion correction can be suggested as the most suitable for theoretical studies of the interaction between molecules and graphene. ${ }^{32}$ Therefore, the all results were obtained with Grimme-corrected PBE. We selected DFT semicore pseudopotential (DSSP) to replace core electrons as a single effective potential. ${ }^{33} \mathrm{~A}$ double numerical plus polarization (DNP) was employed as the basis set. The DNP basis set corresponds to a double- $\zeta$ quality basis set with a p-type polarization functions to hydrogen and d-type polarization functions added to heavier atoms, which was comparable with the Gaussian 6-31G $(\mathrm{d}, \mathrm{p})$ basis set and exhibited a better accuracy. ${ }^{34}$

We applied $5 \times 5 \times 1$ supercell with periodic boundary condition on the $x$ and $y$ axes to model the infinite graphene sheet. The vacuum space of $20 \AA$ was set in the direction normal to the sheets to avoid the interactions between periodic images. A $15 \times 15 \times 1$ mesh of $k$-point and the global orbital cutoff of
$5.0 \AA$ were set in the spin-unrestricted calculations. All atoms are allowed to relax. Convergence in energy, force, and displacement were set at $2 \times 10^{-5} \mathrm{Ha}, 0.004 \mathrm{Ha} \AA^{-1}$, and 0.005 $\AA$, respectively.

We analyzed the adsorption of HCN on hydrogenated graphene (HG). The adsorption energy was obtained from the expression

$$
E_{\mathrm{ads}}=E(\mathrm{HCN} / \mathrm{HG})-E(\mathrm{HG})-E(\mathrm{HCN})
$$

where $E(\mathrm{HCN} / \mathrm{HG})$ is the total energy of $\mathrm{HCN}$ molecule adsorbed on hydrogenated graphene, $E(\mathrm{HG})$ is the total energy of the $\mathrm{HG}$, and $E(\mathrm{HCN})$ is the total energy of a free $\mathrm{HCN}$ molecule, respectively.

\section{Results and discussion}

\subsection{Structures of HG, VHG, BVHG, NVHG, SVHG, and AlVHG}

At first, we considered the optimized geometries of the graphane (HG) and H-vacancy defected graphane (VHG), and the relaxed structures were shown in Fig. 1. In the HG, the bond lengths of C-C and C-H were $1.496 \AA$ and $1.109 \AA$, which was in accord with previous report. ${ }^{35}$ The H-vacancy was created by removing an $\mathrm{H}$ atom from the perfect hydrogenated graphene. Experimentally, the appearance of $\mathrm{H}$-vacancy is very common during the synthetic process, while the graphene is exposed in the hydrogen plasma. After fully relaxation, the $C_{\mathrm{v}}$ atom, which lost a $\mathrm{H}$ atom, exhibited unpaired localized electron due to the
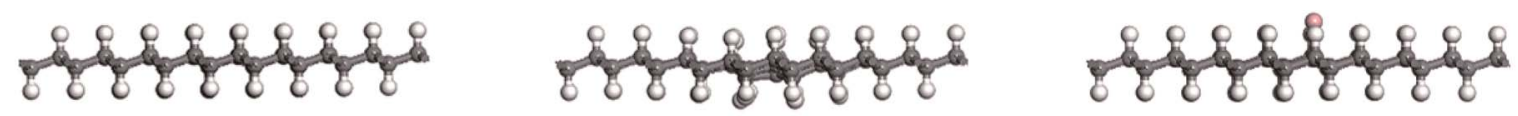

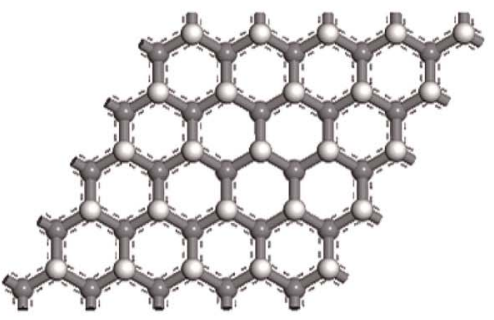

a
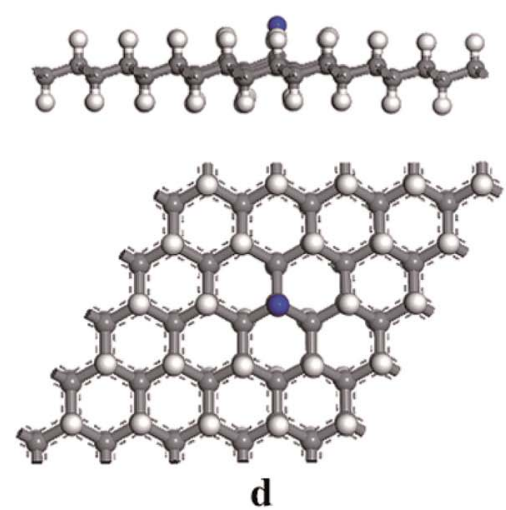
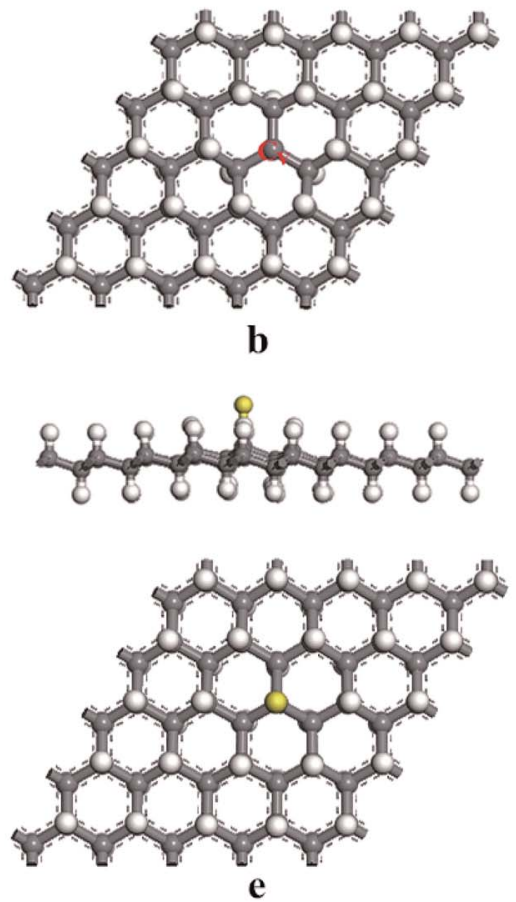
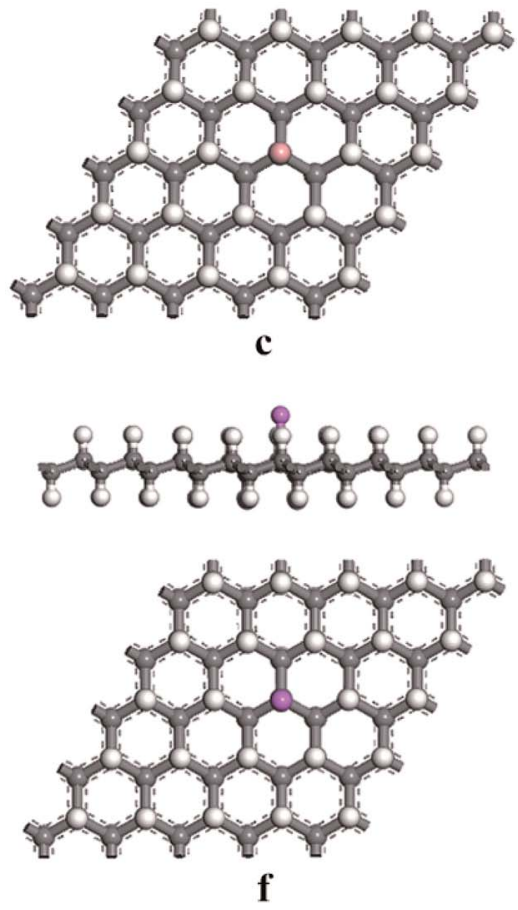

Fig. 1 The optimized structures of (a) HG, (b) VHG, (c) BVHG, (d) NVHG, (e) SVHG, and (f) AlVHG. 
appearance of H-vacancy. As shown in Fig. $1 \mathrm{~b}$, the $C_{\mathrm{v}}$ was labeled. The calculated results indicated that the bond length of C-C around the $C_{\mathrm{v}}$ atom decreased to $1.469 \AA$, which was consistent with previous literatures. ${ }^{25,29}$

According to previous results, the appearance of H-vacancy increased the activity of graphane, and the $C_{\mathrm{v}}$ site exhibited high sensitivity towards foreign atoms or molecules. Thus, we considered the introducing of dopants to VHG, and the dopants included $\mathrm{B}, \mathrm{N}, \mathrm{S}$, and $\mathrm{Al}$ atoms. The optimized doped configurations were performed in Fig. 1c-f. Furthermore, the calculated results suggested that the binding energies of $\mathrm{B}, \mathrm{N}, \mathrm{S}$, and $\mathrm{Al}$ were $2.80 \mathrm{eV}, 2.23 \mathrm{eV}, 2.55 \mathrm{eV}$ and $3.37 \mathrm{eV}$, respectively. The calculated $E_{\mathrm{b}}$ were larger than their cohesive energies, which enhanced the stability of the geometry. To explore the influence of H-vacancy and dopants on the graphane, we performed the band structures of the configurations in Fig. 2, and the spin-up (majority) and spin-down (minority) bands were separated in each case. For HG (Fig. 1a), both band structures were symmetrical, and the band gap was $3.686 \mathrm{eV}$, which was in agreement with the results of Leenaerts et al. ${ }^{36}$ and Islam et al. ${ }^{29}$
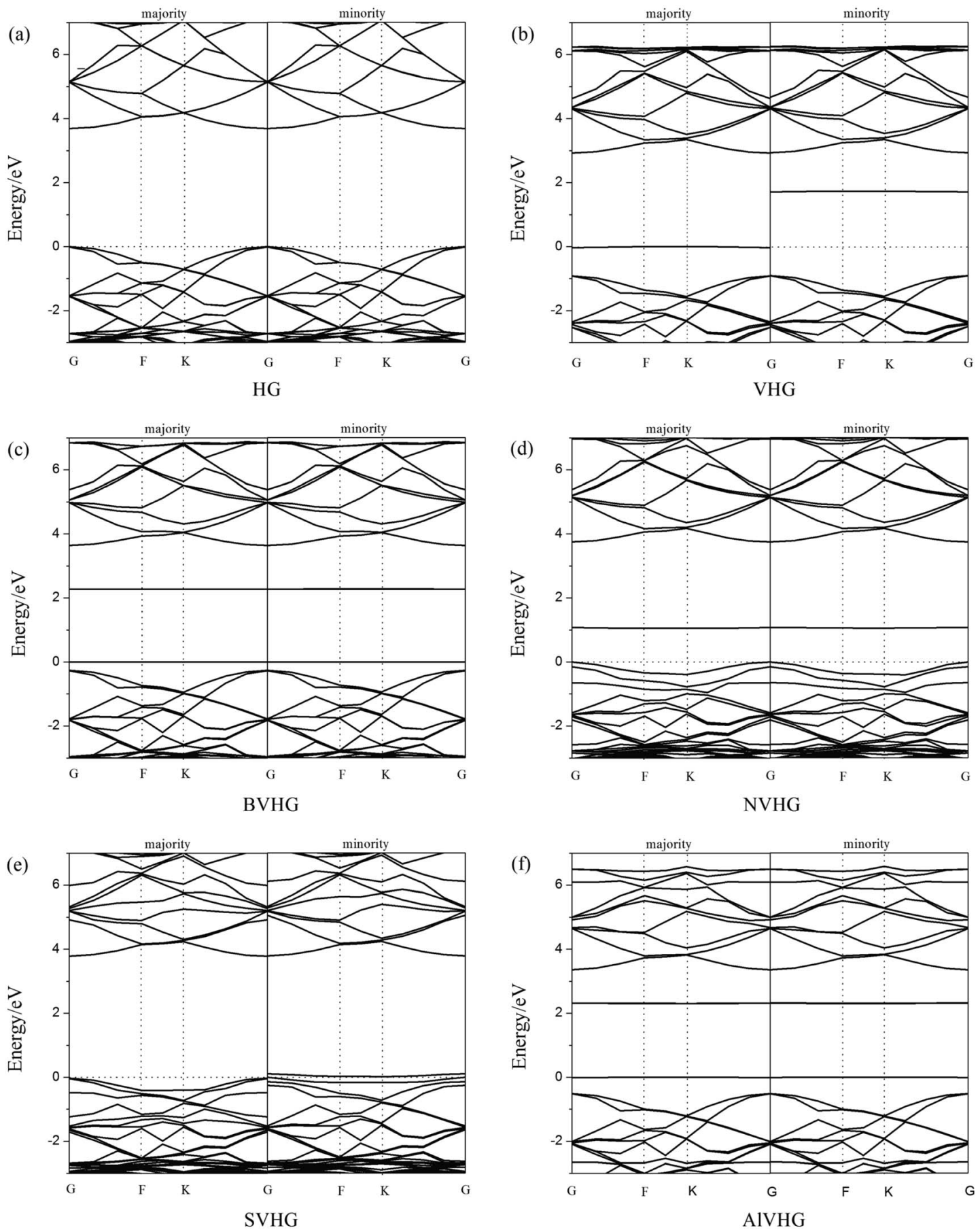

Fig. 2 The band structures of (a) HG, (b) VHG, (c) BVHG, (d) NVHG, (e) SVHG, and (f) AIVHG. 
In the case of VHG, the majority and minority bands were mismatched and the band gap decreased to $1.704 \mathrm{eV}$, which indicated that the structure exhibited net magnetic moments. The appearance of $\mathrm{H}$-vacancy released the $\mathrm{p}$ electron on the $\mathrm{C}$ atom of the graphane and formed a localized state possessing the $0.995 \mu_{\mathrm{B}}$, which induced nonzero magnetization and was consistent with the result of Berashevich et al. ${ }^{23}$ Furthermore, the spin-up bands showed metallic and the spin-down bands exhibited semiconductivity generating a half-metallic character in the band structure of VHG. After the introducing of dopants, the BVHG, NVHG, and AlVHG were nonmagnetic, which were resulted from the unpaired electron of dopants interacted with the localized electron of $C_{\mathrm{v}}$ atom. Moreover, the band gaps for the BVHG, NVHG, and AlVHG were $2.68 \mathrm{eV}, 1.056 \mathrm{eV}$, and $2.296 \mathrm{eV}$, respectively. In the case of SVHG, the Fermi level located within the conduction bands in the spin-down bands, while the spin-up bands maintained a band gap of $2.78 \mathrm{eV}$. Namely, a half-metallic behavior was observed. All in all, the impurity bands appeared around the Fermi level in the doped systems, and then the activity of graphane substrate was expected to be improved to interact with adsorbents.

\subsection{Adsorption of HCN on HG and VHG}

First, the adsorption of HCN molecule on the surface of HG and VHG were investigated. In order to explore the most stable adsorption structure, the initial adsorption structures for HCN molecule were considered as follows: the $\mathrm{H}$ atom close to graphane with the HCN molecule perpendicular to the surface ( $\mathrm{H}$ site), the $\mathrm{C}$ atom close to graphane with the HCN molecule parallel to the surface ( $\mathrm{C}$ site), and the $\mathrm{N}$ atom close to graphane with the HCN molecule perpendicular to the surface ( $\mathrm{N}$ site). The optimized adsorption systems of $\mathrm{H}-$ $\mathrm{HCN} / \mathrm{HG}, \mathrm{C}-\mathrm{HCN} / \mathrm{HG}$, and N-HCN/HG were performed in Fig. 3. Meanwhile, for the "C site" in VHG, the $\mathrm{C}$ atom of HCN molecule was close to the $C_{\mathrm{v}}$ atom of VHG. After optimization, the adsorption structures of HCN on VHG were also showed in Fig. 4. Furthermore, the calculated bond lengths of HCN molecule, adsorption distance between the $\mathrm{HCN}$ and the graphane, and the adsorption energy were summarized in Table 1 . In the cases of $\mathrm{HCN} / \mathrm{HG}$, the adsorption energies were $-0.019 \mathrm{eV},-0.021 \mathrm{eV}$, and $-0.011 \mathrm{eV}$, respectively. Compared with $\mathrm{HCN} / \mathrm{HG}$, the
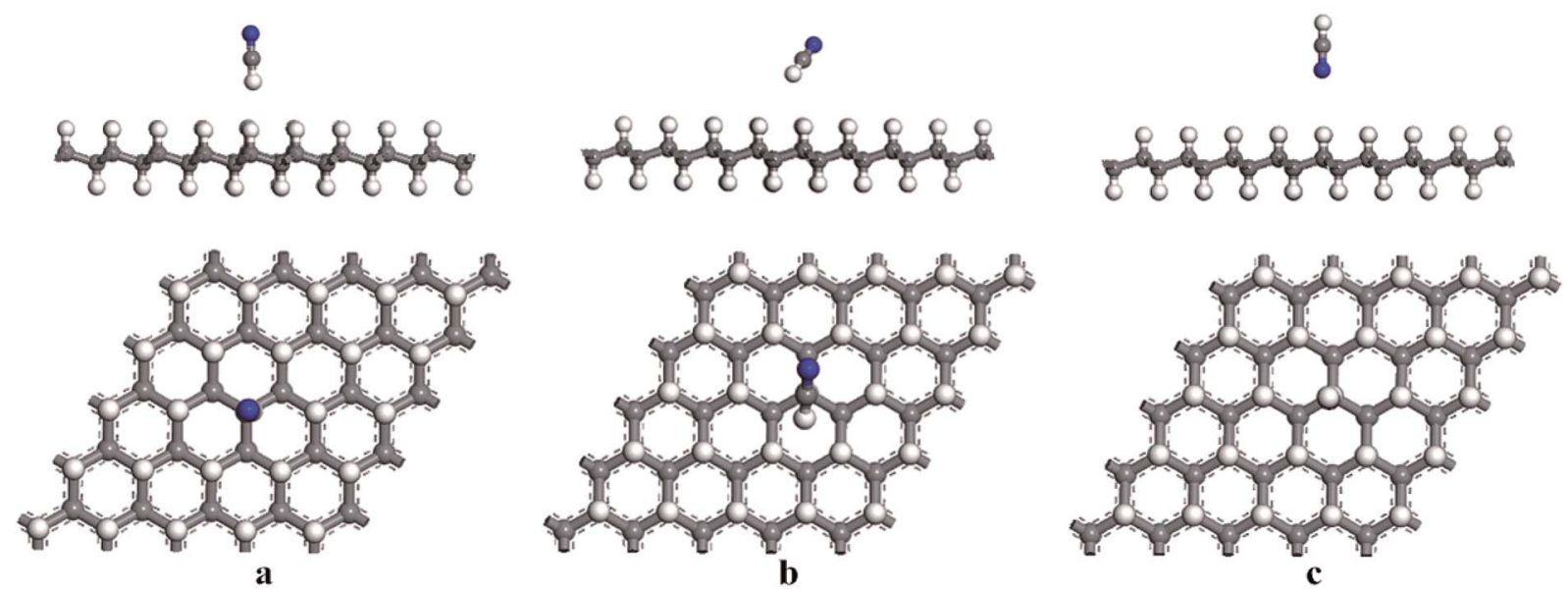

Fig. 3 The optimized structures of (a) $\mathrm{H}-\mathrm{HCN} / \mathrm{HG}$, (b) $\mathrm{C}-\mathrm{HCN} / \mathrm{HG}$, and (c) $\mathrm{N}-\mathrm{HCN} / \mathrm{HG}$.
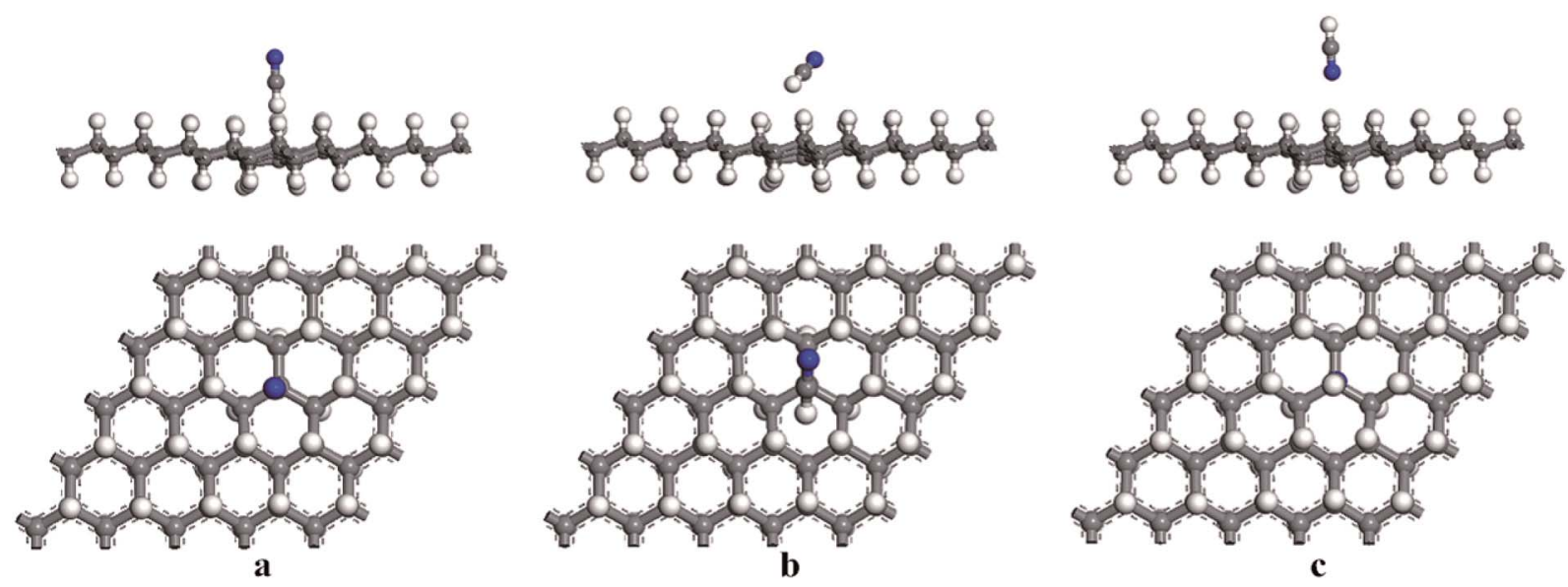

Fig. 4 The optimized structures of (a) $\mathrm{H}-\mathrm{HCN} / \mathrm{VHG}$, (b) $\mathrm{C}-\mathrm{HCN} / \mathrm{VHG}$, and (c) N-HCN/VHG. 
Table 1 Summary of the results for the $\mathrm{HCN}$ molecule adsorbed on pure graphane $(\mathrm{HG})$ and $\mathrm{H}$-vacancy defected graphane (VHG). The properties listed are as follows: the distance between the $\mathrm{H}$ and $\mathrm{C}$ atom of $\mathrm{HCN}$ molecule $\left(d_{1}\right)$, the distance between the $\mathrm{N}$ and $\mathrm{C}$ atom of $\mathrm{HCN}$ molecule $\left(d_{2}\right)$, the distance between the molecule $\mathrm{HCN}$ and graphane surface $\left(d_{M-G}\right)$, the adsorption energy $\left(E_{\text {ads }}\right)$

\begin{tabular}{llllll}
\hline System & Site & $d_{1}(\AA)$ & $d_{2}(\AA)$ & $d_{\text {M-G }}(\AA)$ & $E_{\text {ads }}(\mathrm{eV})$ \\
\hline HCN/HG & $\mathrm{H}$ & 1.078 & 1.163 & 3.239 & -0.019 \\
& $\mathrm{C}$ & 1.077 & 1.163 & 3.207 & -0.021 \\
& $\mathrm{~N}$ & 1.076 & 1.162 & 3.686 & -0.011 \\
HCN/VHG & $\mathrm{H}$ & 1.087 & 1.163 & 3.583 & -0.048 \\
& $\mathrm{C}$ & 1.077 & 1.162 & 2.568 & -0.088 \\
& $\mathrm{~N}$ & 1.076 & 1.162 & 3.794 & -0.027
\end{tabular}

adsorption of HCN molecule on VHG exhibited higher stability. The results indicated that the C-HCN/VHG was the most stable system, of which the $E_{\text {ads }}$ was $-0.088 \mathrm{eV}$. The larger adsorption energies indicated that the appearance of $\mathrm{H}$-vacancy in graphane improved the activity of graphane substrate. For the two adsorption substrates, the "C site" systems exhibited most stability than the other two adsorption structures. However, the relative large adsorption distance and small adsorption energies showed that the interaction between the HCN molecule and the HG and VHG surface were physisorption.
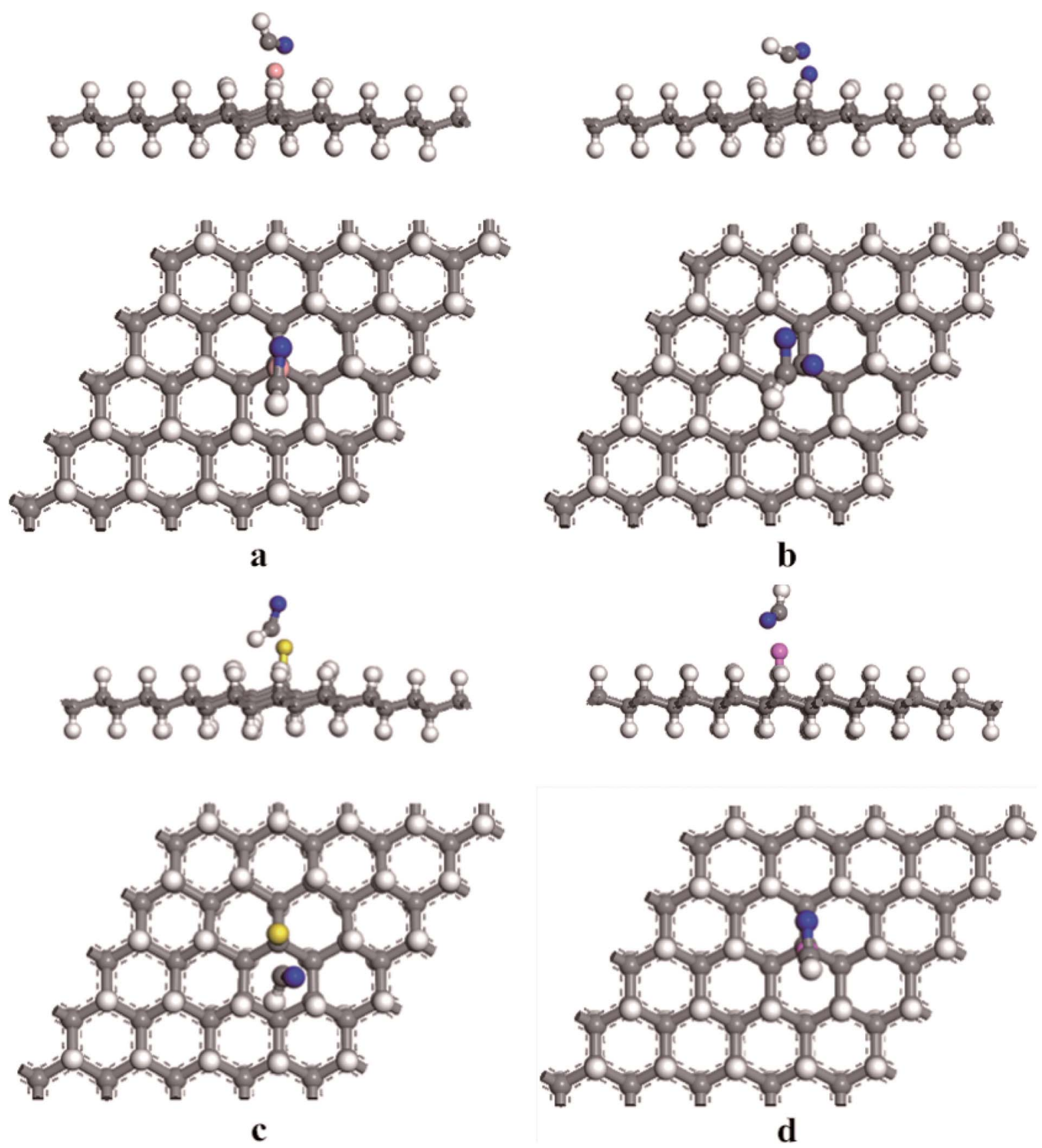

Fig. 5 The optimized adsorption structures of (a) HCN/BVHG, (b) HCN/NVHG, (c) HCN/SVHG, and (d) HCN/AIVHG. 


\subsection{Adsorption of HCN on BVHG, NVHG, SVHG, and AlVHG}

According to the discussion in 3.2, for the adsorption of HCN on HG and VHG, the initial adsorption system for "C site" was the most energetically favorable system. Thus, we set the HCN molecule parallel to the doped graphane (BVHG, NVHG, SVHG, and AlVHG) with the $\mathrm{C}$ atom close to the dopants in the adsorption configurations. The optimized configurations of HCN/BVHG, HCN/NVHG, HCN/SVHG, and HCN/AlVHG were shown in Fig. 5. Furthermore, the corresponding parameters detailedly describing the adsorption systems were summarized in Table 2. As shown in Fig. 5, the HCN molecule did not maintain the linear bond structure after interacting with the doped graphane. The bond lengths of $\mathrm{C}$ atom of HCN molecule and the dopants were $1.406 \AA$, $1.381 \AA$, $1.796 \AA$, and $1.867 \AA$ for the adsorption complexes, respectively. As performed in Table 2 , the HCN/NVHG exhibited the largest $E_{\text {ads }}$ with the value of $-1.144 \mathrm{eV}$. Meanwhile, the adsorption energies varied from $-0.115 \mathrm{eV}$ to $-1.144 \mathrm{eV}$, which were all larger than that of $\mathrm{HG}$ and VHG. The results suggested that the introducing of dopants (B, N, S, and Al) improved the sensitivity of graphane to HCN gas molecules. Moreover, the charge transfer of HCN molecule was analyzed by Hirshfeld method which seems to be more reliable and yield chemically meaningful charges in comparison with Mulliken method. ${ }^{37,38}$ Take the case of HCN/NVHG as example, the N-dopant accepted 0.359 e and the HCN molecule lost 0.125 e, which indicated that some electrons transferred from HCN molecule and graphane substrate to the N-dopant, illustrating

Table 2 Summary of the results for the HCN molecule adsorbed on VHG with dopants (B, N, S, Al). The properties listed are as follows: the distance between the $\mathrm{H}$ and $\mathrm{C}$ atom of $\mathrm{HCN}$ molecule $\left(d_{1}\right)$, the distance between the $\mathrm{N}$ and $\mathrm{C}$ atom of $\mathrm{HCN}$ molecule $\left(d_{2}\right)$, the bond length of $C_{v}$ atom and the dopant $\left(d_{3}\right)$, the distance between the molecule $C$ atom of $\mathrm{HCN}$ molecule and dopant $\left(d_{4}\right)$, the adsorption energy $\left(E_{\text {ads }}\right)$, the band gap of adsorption system $\left(E_{\mathrm{g}}\right)$, and the charge transfer of the $\mathrm{HCN}$ molecule $(Q)$

\begin{tabular}{llllllll}
\hline System & $d_{1}(\AA)$ & $d_{2}(\AA)$ & $d_{3}(\AA)$ & $d_{4}(\AA)$ & $E_{\text {ads }}(\mathrm{eV})$ & $E_{\mathrm{g}}(\mathrm{eV})$ & $Q(\mathrm{e})$ \\
\hline HCN/BVHG & 1.095 & 1.315 & 1.575 & 1.406 & -1.040 & 2.400 & -0.121 \\
HCN/NVHG & 1.093 & 1.258 & 1.496 & 1.381 & -1.144 & 2.426 & 0.125 \\
HCN/SVHG & 1.101 & 1.254 & 1.896 & 1.796 & -0.115 & 0.821 & -0.036 \\
HCN/AlVHG & 1.090 & 1.300 & 2.015 & 1.867 & -0.412 & 2.406 & -0.394
\end{tabular}

that the dopants can facilitate the interaction between the HCN molecule and the graphane substrate.

As shown in Table 2, we calculated the band gaps of the adsorption systems. The adsorption of HCN induced a band open to the configurations. In case of NVHG, the band gap changed from $1.056 \mathrm{eV}$ to $2.426 \mathrm{eV}$ by the interaction with the HCN molecule. For other cases, the band gaps of BVHG (2.268 $\mathrm{eV}$, SVHG $(0.020 \mathrm{eV})$, and AlVHG $(2.296 \mathrm{eV})$ increased to $2.400 \mathrm{eV}, 0.821 \mathrm{eV}$, and $2.406 \mathrm{eV}$, respectively. It is well known the change of $E_{\mathrm{g}}$ induces the electric conductivity change of graphane substrate according to the following equation: ${ }^{39}$

$$
\sigma \propto \exp \left(\frac{-E_{\mathrm{g}}}{2 k T}\right)
$$

where $\sigma$ is the electric conductivity of the configurations, $k$ is the Boltzmann's constant, and $T$ is the thermodynamic temperature. Therefore, the change of conductance of graphane substrate can be seen as signal to detect $\mathrm{HCN}$ gas molecules.

Moreover, the adsorptions of $\mathrm{H}_{2} \mathrm{O}$ and $\mathrm{NO}_{2}$ molecules on the modified graphane substrates were investigated, aiming to explore the selectivity of the structures used as sensors to detect the HCN gas. The most stable final adsorption configurations were performed in Fig. 6 and 7. The adsorption energies of the $\mathrm{H}_{2} \mathrm{O}$ molecules on BVHG, NVHG, SVHG, and AlVHG were $-0.012 \mathrm{eV},-0.030 \mathrm{eV},-0.007 \mathrm{eV}$, and $-0.025 \mathrm{eV}$, respectively. The interaction distances were labeled in Fig. 6. According to the calculated results, the small adsorption energies and large bond distances indicated that the adsorption were physisorptions. Furthermore, the modified substrates were slightly changed by the adsorption of $\mathrm{H}_{2} \mathrm{O}$ molecule. Compared with the case of $\mathrm{HCN}$, the presence of $\mathrm{H}_{2} \mathrm{O}$ molecules cannot interfere in the detection of HCN.

The most stable structure of the $\mathrm{NO}_{2}$ adsorption systems was $\mathrm{NO}_{2} /$ AlVHG, and the $E_{\text {ads }}$ was $-0.105 \mathrm{eV}$ and the band gap changed by $0.076 \mathrm{eV}$. The interaction was somewhat strong, though the change of band gap was smaller than the case of HCN. Therefore, we think that the presence of $\mathrm{NO}_{2}$ gas molecule may interfere in detecting of the HCN gas.

\subsection{Electronic properties}

To gain more insight into the effect of the H-vacancy and dopants on the adsorption of HCN molecule on the graphane,

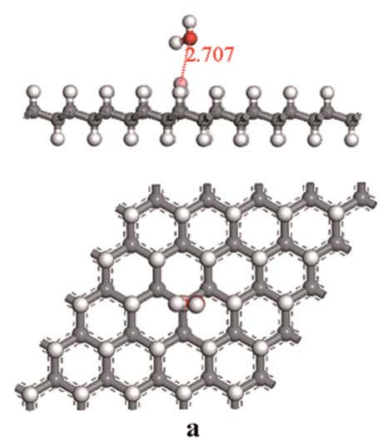

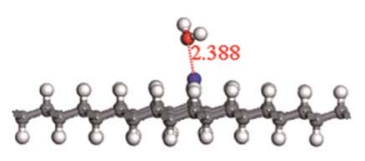

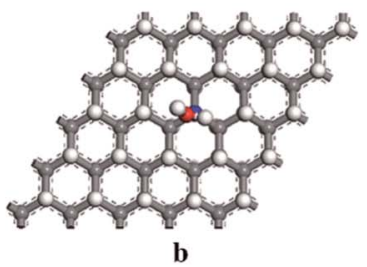

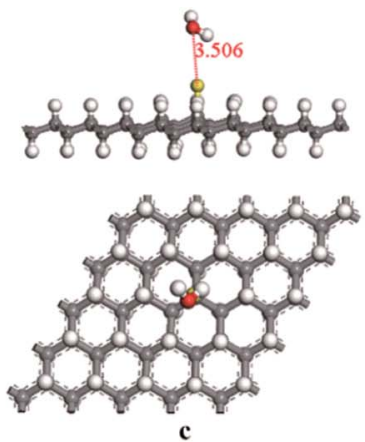

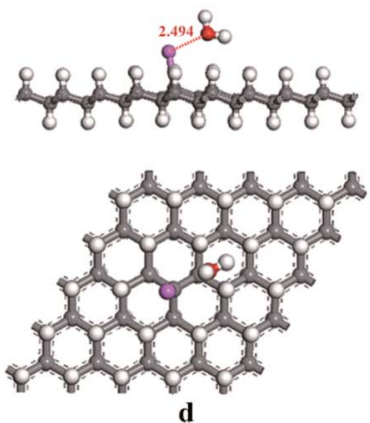

Fig. 6 The optimized adsorption structures of (a) $\mathrm{H}_{2} \mathrm{O} /$ BVHG, (b) $\mathrm{H}_{2} \mathrm{O} / \mathrm{NVHG}$, (c) $\mathrm{H}_{2} \mathrm{O} / \mathrm{SVHG}$, and (d) $\mathrm{H}_{2} \mathrm{O} / \mathrm{AlVHG}$. 

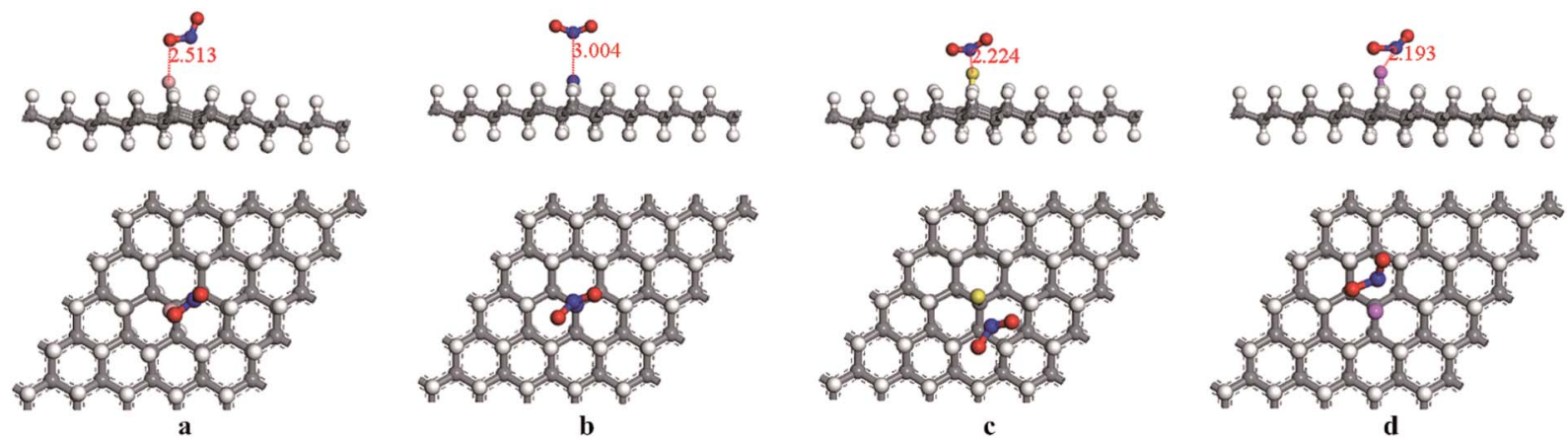

Fig. 7 The optimized adsorption structures of (a) $\mathrm{NO}_{2} / \mathrm{BVHG}$, (b) $\mathrm{NO}_{2} / \mathrm{NVHG}$, (c) $\mathrm{NO}_{2} / \mathrm{SVHG}$, and (d) $\mathrm{NO}_{2} / \mathrm{AlVHG}$.

we investigated the change of electronic structures by performing the total density of states (TDOS) and spin polarized density of states (PDOS) of the adsorption complexes in Fig. 813. As shown in Fig. 8 and 9, the DOS showed that the HCN/HG exhibited nonmagnetic, while the HCN/VHG exhibited magnetic characters. The PDOS of $C_{\mathrm{v}}$ atom in Fig. 9a showed that the H-vacancy induced the localized electron of $C_{\mathrm{v}}$ showed a obvious half-metallic behavior and complete spin-polarized states around the Fermi level. The PDOS plots was consistent with the band structures of VHG performed in Fig. 2b, which implied that the appearance of H-vacancy produced significant change in the electronic and magnetic properties of HG.
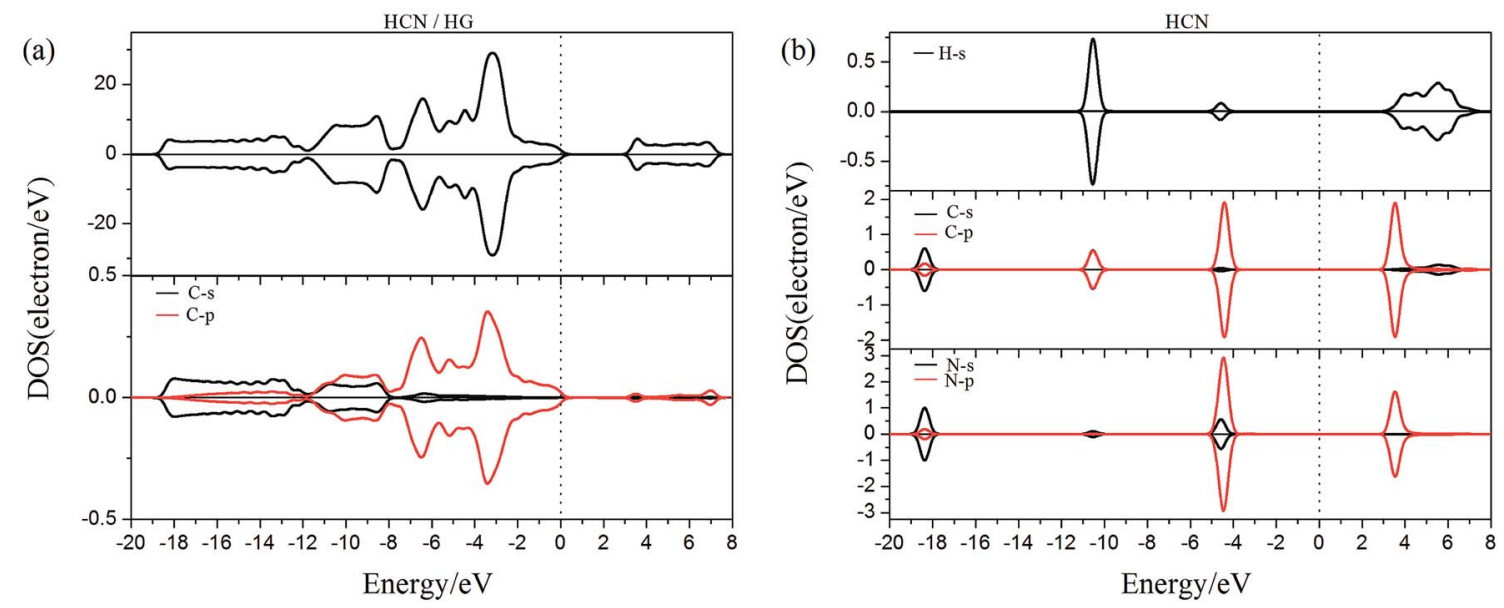

Fig. 8 The PDOS of configurations of (a) HCN/HG and (b) HCN molecule.
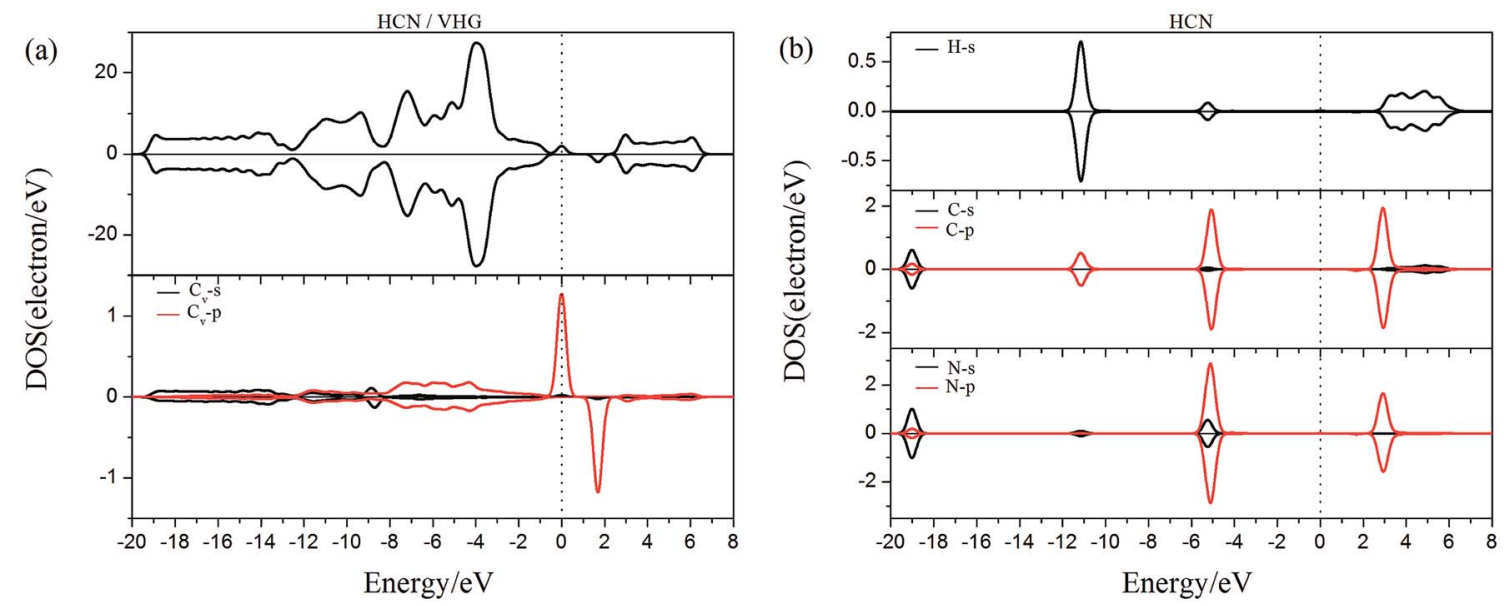

Fig. 9 The PDOS of configurations of (a) HCN/VHG and (b) HCN molecule. 

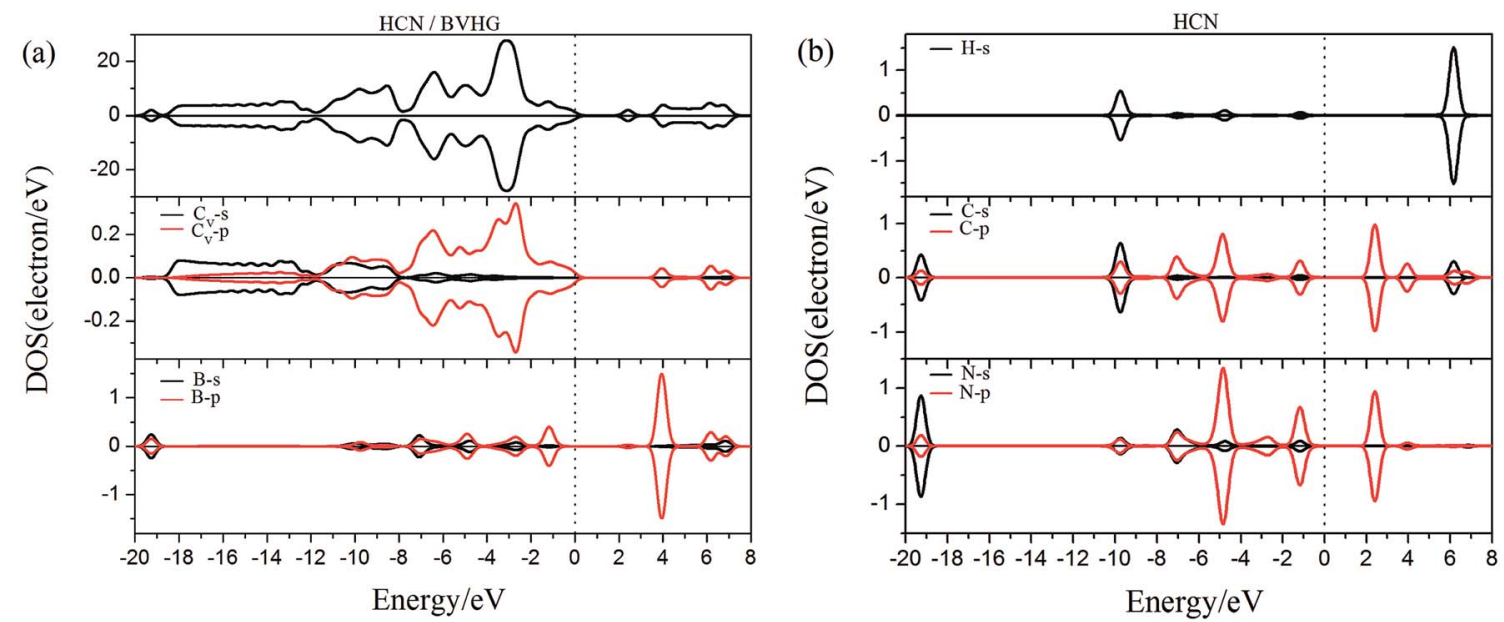

Fig. 10 The PDOS of configurations of (a) HCN/BVHG and (b) HCN molecule.
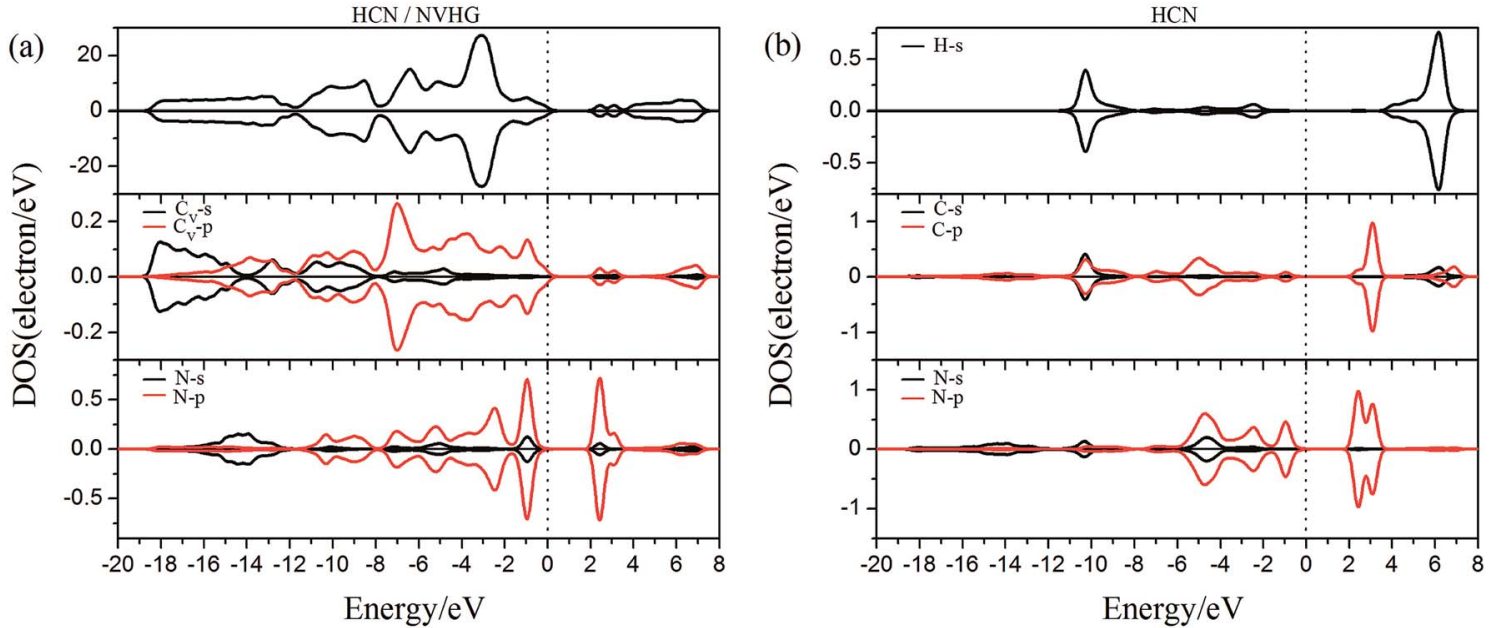

Fig. 11 The PDOS of configurations of (a) HCN/NVHG and (b) HCN molecule.

Furthermore, a few overlaps between the HCN molecule and the HG and VHG substrates, which indicated that the interaction were very weak.
As performed in Fig. 10a-13a, a large area of hybridization between the dopants $(\mathrm{B}, \mathrm{N}, \mathrm{S}, \mathrm{Al})$ and the $C_{\mathrm{v}}$ atom was observed below and above the Fermi level. It was well agreement with the
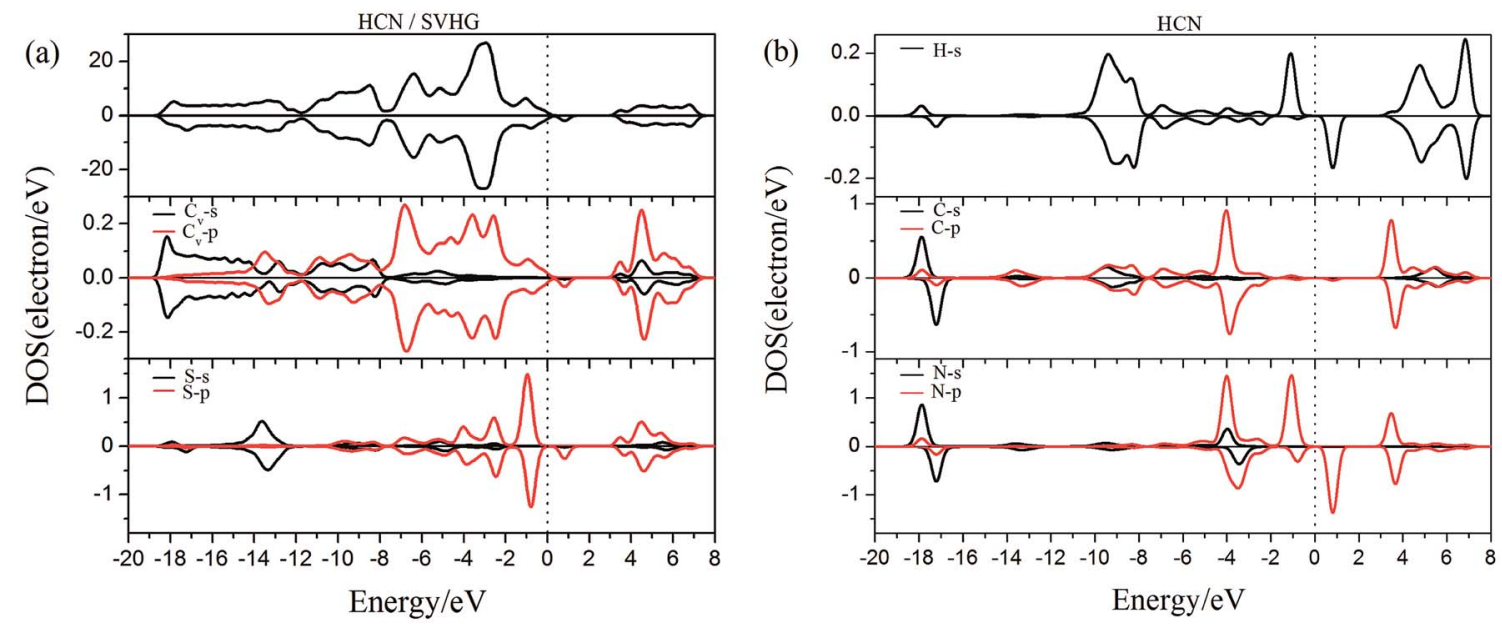

Fig. 12 The PDOS of configurations of (a) HCN/SVHG and (b) HCN molecule. 

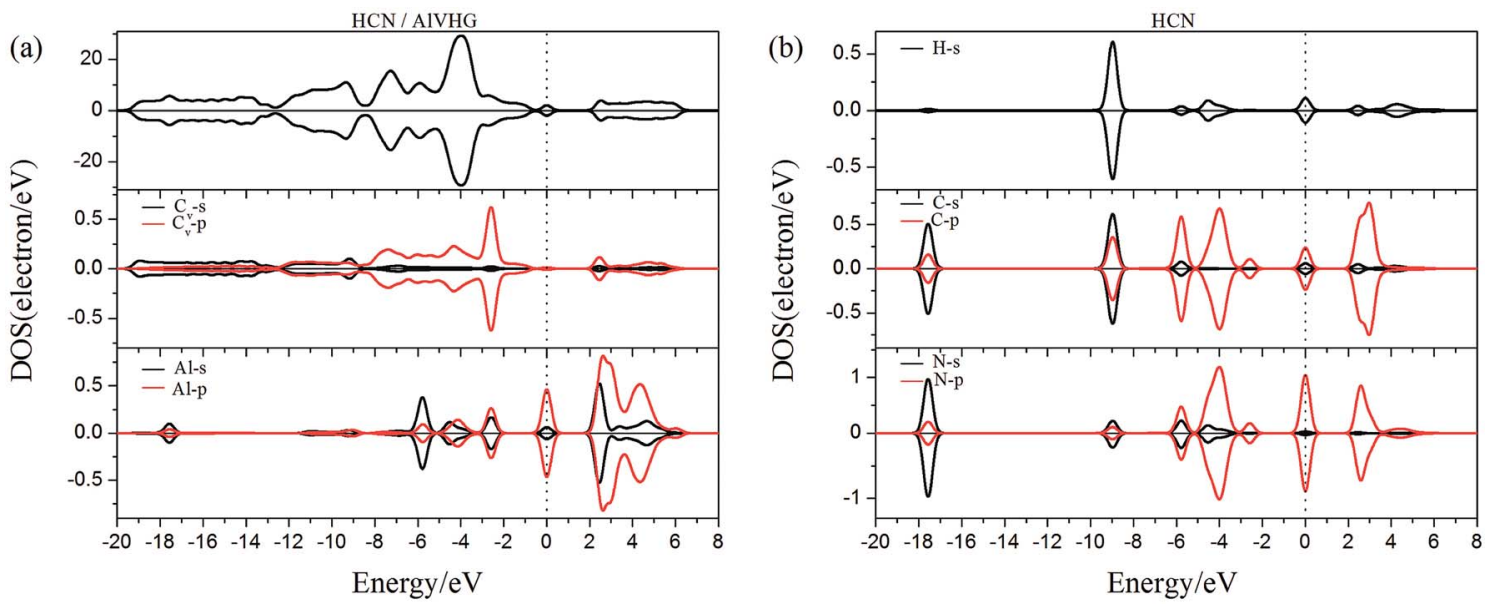

Fig. 13 The PDOS of configurations of (a) HCN/AIVHG and (b) HCN molecule.

large binding energies of the VHG for the dopants and chemical bonds were formed between the dopants and $C_{\mathrm{v}}$ atom. Furthermore, the HCN/BVHG, HCN/NVHG, and HCN/AlVHG exhibited nonmagnetic according to the symmetry of spin channels, while the HCN/SVHG exhibited net magnetic moment. Compared with the structures without absorbing HCN molecules, the results suggested that the adsorption of HCN hardly influence the magnetic character of the VHG with dopants. In the case of HCN/NVHG, the most stable adsorption complex, the obvious hybridization between the N-2p orbital of substrate and C-2p orbital of HCN molecule below the Fermi level indicated that their strong interaction, which was responsible for the large adsorption energy of HCN molecule. As shown in Fig. 12, the spin-up and spin-down channels of HCN molecule became asymmetric and then the HCN/SVHG configuration exhibited magnetic property with the net moment of $0.998 \mu_{\mathrm{B}}$. Furthermore, the spin-down channel exhibited nearly zero-gap semiconductivity, while the spin-up channel showed non-zero DOS at the Fermi level. The result indicated that the HCN/SVHG system maintained the property of half-metallicity. Based on the above discussions, the HCN molecule on the doped HG exhibited higher adsorption stability than that of HG and VHG. The PDOS plots indicated that the large hybridization between the HCN molecule and dopants, which meant that the strong adsorption of HCN on the doped substrates.

\section{Conclusions}

Based on the first-principles calculations, we investigated the stable structures of hydrogenated graphene (graphane) doped with $\mathrm{B}, \mathrm{N}, \mathrm{S}$, and $\mathrm{Al}$, which used as potential gas sensing device to detect HCN molecules. The results indicated that the appearance of H-vacancy improved the activity of graphane and induced a half-metallicity behavior. However, the weak interaction between the HCN molecule and the HG and VHG substrates illustrated the adsorption was physisorption. After introducing the dopants, the chemical bonds formed between the dopants and the $C_{\mathrm{v}}$ atom. Moreover, the PDOS results suggested that the impurity peaks appeared around the Fermi level, which indicated that the dopants improved the sensitivity to adsorbents. The large adsorption energies and small bond distance indicated that the adsorption of HCN on doped HG substrates transferred into chemisorption. Furthermore, the adsorption of HCN induced a band gap open on the BVHG, NVHG, SVHG, and AlVHG systems, which could change the conductance of configurations. Therefore, the controlling of electronic and magnetic properties of graphane by the adsorption of HCN molecules is expected to provide meaningful approach for designing HCN gas sensing device.

\section{Conflicts of interest}

There are no conflicts to declare.

\section{Acknowledgements}

This work is supported by the National Natural Science Foundation of China (NSFC, Grant No. 11604080, 11404096, U1404609, 11304080, 11547153) and the Innovation Team of Henan University of Science and Technology (No. 2015XTD001).

\section{References}

1 Y. Qin, X. Sun, X. Li and M. Hu, Sens. Actuators, B, 2012, 162, 244-250.

2 S. Capone, A. Forleo, L. Francioso, R. Rella, P. Siciliano, J. Spadavecchia, D. S. Presicce and A. M. Taurino, ChemInform, 2004, 35, 1335-1348.

3 F. Hossein-Babaei and A. Amini, Sens. Actuators, B, 2012, 166, 419-425.

4 R. Wang, D. Zhang, Y. Liu and C. Liu, Nanotechnology, 2009, 20, 505704 .

5 A. Zabardasti, A. Kakanejadifard and M. Ghasemian, Struct. Chem., 2012, 23, 1155-1161.

6 N. Barsan, D. Koziej and U. Weimar, Sens. Actuators, B, 2007, 121, 18-35. 
7 A. Kolmakov and M. Moskovits, Annu. Rev. Mater. Res., 2004, 35, 151-180.

8 K. S. Novoselov, A. K. Geim, S. V. Morozov, D. Jiang, Y. Zhang, S. V. Dubonos, I. V. Grigorieva and A. A. Firsov, Science, 2004, 306, 666.

9 S. Stankovich, D. A. Dikin, G. H. B. Dommett, K. M. Kohlhaas, E. J. Zimney, E. A. Stach, R. D. Piner, S. T. Nguyen and R. S. Ruoff, Nature, 2006, 442, 282-286.

10 M. Sun and Y. Peng, Appl. Surf. Sci., 2014, 307, 158-164.

11 Z. Dai and Y. Zhao, Appl. Surf. Sci., 2014, 305, 382-385.

12 Y. Tang, W. Chen, C. Li, L. Pan, X. Dai and D. Ma, Appl. Surf. Sci., 2015, 342, 191-199.

13 J. W. Gu, C. Liang, X. Zhao, B. Gan, H. Qiu, Y. Guo, X. Yang, Q. Zhang and D. Y. Wang, Compos. Sci. Technol., 2017, 139, 83-89.

14 X. Zhang, L. Yu, Y. Gui and W. Hu, Appl. Surf. Sci., 2016, 367, 259-269.

15 A. S. Rad and E. Abedini, Appl. Surf. Sci., 2016, 360, 10411046.

16 B. S. Li, P. W. Yong and K. D. Hai, Appl. Surf. Sci., 2015, 329, 330-336.

17 D. C. Elias, R. R. Nair, T. M. G. Mohiuddin, S. V. Morozov, P. Blake, M. P. Halsall, A. C. Ferrari, D. W. Boukhvalov, M. I. Katsnelson and A. K. Geim, Science, 2009, 323, 610-613.

18 H. Şahin, C. Ataca and S. Ciraci, Appl. Phys. Lett., 2009, 95, 222510.

19 T. Hussain, B. Pathak, M. Ramzan, T. A. Maark and R. Ahuja, Appl. Phys. Lett., 2012, 100, 302-304.

20 S. Haldar, D. G. Kanhere and B. Sanyal, Phys. Rev. B: Condens. Matter Mater. Phys., 2012, 85, 155426.

21 T. Hussain, A. D. Sarkar and R. Ahuja, Appl. Phys. Lett., 2012, 101, 103907.

22 C. Zhou, S. Chen, J. Lou, J. Wang, Q. Yang, C. Liu, D. Huang and T. Zhu, Nanoscale Res. Lett., 2014, 9, 26.
23 J. Berashevich and T. Chakraborty, Phys. Rev. B: Condens. Matter Mater. Phys., 2010, 82, 876-877.

24 B. Julia and C. Tapash, Nanotechnology, 2010, 21, 355201.

25 R. E. Mapasha, M. P. Molepo and N. Chetty, Phys. E, 2016, 79, 52-58.

26 C. K. Yang, Carbon, 2010, 48, 3901-3905.

27 T. Hussain, P. Panigrahi and R. Ahuja, Phys. Chem. Chem. Phys., 2014, 16, 8100-8105.

28 H. Tanveer, P. Puspamitra and A. Rajeev, Nanotechnology, 2014, 25, 325501.

29 M. S. Islam, T. Hussain, G. S. Rao, P. Panigrahi and R. Ahuja, Sens. Actuators, B, 2016, 228, 317-321.

30 B. Delley, J. Chem. Phys., 2000, 113, 7756-7764.

31 J. P. Perdew, K. Burke and M. Ernzerhof, Phys. Rev. Lett., 1996, 77, 3865-3868.

32 V. A. Basiuk and L. V. Henaoholguín, J. Comput. Theor. Nanosci., 2014, 11, 1609-1615.

33 D. J. Chadi, Phys. Rev. B: Condens. Matter Mater. Phys., 1976, 13, 5188-5192.

34 B. Delley, Phys. Rev. B: Condens. Matter Mater. Phys., 2002, 66, 155125.

35 J. O. Sofo, A. S. Chaudhari and G. D. Barber, Phys. Rev. B: Condens. Matter Mater. Phys., 2007, 75, 153401.

36 O. Leenaerts, H. Peelaers, A. D. Hernández-Nieves, B. Partoens and F. M. Peeters, Phys. Rev. B: Condens. Matter Mater. Phys., 2010, 82, 195436.

37 C. Fonseca Guerra, J. W. Handgraaf, E. J. Baerends and F. M. Bikelhaupt, J. Comput. Chem., 2004, 25, 189.

38 A. E. Clark, J. L. Sonnenberg, P. J. Hay and R. L. Martin, Chem. Phys., 2004, 121, 2563-2570.

39 S. S. Li, Semiconductor physical electronics, Springer, USA, 2nd edn, 2006. 